J. Appl. Cryst. (1990). 23, 21-25

\title{
Measurement of Anisotropic Diffuse Scattering with Synchrotron Radiation
}

\author{
By E. Rosshirt AND F. FreY \\ Institut für Kristallographie und Mineralogie, LMU, Theresienstrasse 41, 8000 München 2, \\ Federal Republic of Germany \\ V. KUPCIK \\ Institut für Mineralogie und Kristallographie, Universität, V. M. Goldschmidtstrasse 1, 3400 Göttingen, \\ Federal Republic of Germany \\ AND G. MIEHE \\ Instititut für Kristallographie, Universität, Senckenberg-Anlage 30, 6000 Frankfurt, \\ Federal Republic of Germany
}

(Received 31 August 1988; accepted 12 September 1989)

\begin{abstract}
Anisotropic diffuse scattering of the ID conductor $\mathrm{K}$-hollandite has been measured at a synchrotron source and compared with measurements with a rotating anode. The intensity gain of more than one order of magnitude and the ability to adjust the wavelength provide the counting statistics in a few hours that are necessary for a quantitative analysis of diffuse phenomena. The main advantage of a synchroton source is the high resolution throughout reciprocal space (low and high $Q$ ). Thus, commensurate/incommensurate positions of diffuse layers may be distinguished, weak diffuse maxima become detectable and small peak shifts of diffuse modulations clearly visible. Correlation functions may be determined directly from the diffuse profiles without tedious resolution corrections. These features are essential for a dedicated instrument at a storage ring.
\end{abstract}

\section{Introduction}

Diffuse scattering from crystals indicates deviations from the strict periodicity in space and time at least in an averaged sense. Cooperative disorder phenomena cause diffuse scattering which is usually anisotropically distributed in reciprocal space and weaker than Bragg scattering by some orders of magnitude. In consequence, for quantitative analysis, high primary intensity, low background scattering, an optimum energy (wavelength), good resolution and well adapted scanning procedures are essential. Previous reports on diffuse scattering from local atomic arrangements using synchrotron radiation are given by Matsubara \& Georgopoulos (1985) and Ohshima, Harada, Morinaga, Georgopoulos \&

0021-8898/90/010021-05\$03.00
Cohen (1986). Most of their statements are generally valid. Additional aspects relating to the measurement of highly anisotropic diffuse phenomena are the subject of this paper.

As an example we use the ID ionic conductor $\mathrm{K}$-hollandite $\mathrm{K}_{1.54} \mathrm{Mg}_{0.77} \mathrm{Ti}_{7 \cdot 23} \mathrm{O}_{16}$, which has been investigated by $\mathrm{X}$-ray and neutron scattering (Rosshirt, 1988; Rosshirt, Frey, Boysen, Eckold \& Steigenberger, 1988; Rosshirt, Frey \& Boysen, 1988). The structure consists of a tetragonal framework of $\mathrm{Ti} / \mathrm{MgO}_{6}$ octahedra (space group $14 / m, a=10 \cdot 13, c$ $=2.97 \AA$ ). The $\mathrm{K}^{+}$ions are embedded in channels parallel to the $c$ axis substoichiometrically. Their ordering behaviour along $\mathbf{c}$ is responsible for a diffuse layer-line system perpendicular to $\mathbf{c}^{*}$. Broad maxima within the diffuse layers indicate lateral correlations between the chains or other structural elements. The main aims of the synchrotron experiments were to determine the exact positions of the diffuse layers, their widths and profiles, and to define the modulations within the layers: positions, extinction rules, width of the maxima parallel and perpendicular to $c^{*}$ and relative intensities.

\section{Experimental}

The experiments were performed at HASYLABDORIS $(5 \cdot 1 \mathrm{GeV}, 30-50 \mathrm{~mA})$ on the five-circle diffractometer (Kupcik, Wulf, Wendschuh, Wolf \& Pähler, 1983; Kupcik, Wendschuh-Josties, Wolf \& Wulf, 1986) using a $\mathrm{Ge}(111)$ double monochromator. For comparison the same phenomena were studied also with a rotating anode $(6 \mathrm{~kW}$; Mo $K \alpha)$ with a Weissenberg diffractometer with focusing $\mathrm{Si}(111)$ monochromator. Whereas the five-circle diffractometer is not optimized for diffuse scattering work, (C) 1990 International Union of Crystallography 
the laboratory equipment is highly efficient in this type of study. Owing to a large focusing angle $\left(1-2^{\circ}\right)$, a relatively extended crystal - in one dimension - can be used without loss of resolution.

\section{Results and discussion}

A $(10 \zeta)$ scan perpendicular to the diffuse layers (synchrotron) is shown in Fig. 1. The strongest layer is clearly visible at $l=0.78$ as a local maximum, with a peak-to-background ratio of about 2:1. The ratio of the integrated intensity of this peak - integrated over $\zeta$ - and the integrated intensity of a weak Bragg reflection (703) is about $1: 10^{3}$. The diffuse layer at $l$ $=1.78$ cannot be separated from the background due to a local intensity minimum at $(1,0,1 \cdot 78)$. On the Weissenberg diffractometer a 15 -fold increase in counting time is necessary for comparable counting statistics: the primary intensity which is lower by a factor of $10^{6}(0.71 \AA)$ is partly compensated for by a large solid angle (18 compared with $0.003 \mathrm{mrad}^{2}$ ). The peak-to-background ratio is similar, the resolution is worse (see below).

The increasing background in the low-angle range (Fig. 1), due the monochromator unit and the air scattering, prevents the observation of weak diffuse layers if any are present. Their existence would help to decide between different disorder models. It is not possible to subtract the parasitic scattering by an 'empty scan' or to obtain information from other parts of reciprocal space (cf. Ohshima et al., 1986).

The selection of an optimum wavelength with respect to scattering power, absorption and fluorescence scattering is particularly valuable in diffuse scattering work. When $\lambda$ was changed from 0.4 to $1.2 \AA$ in steps of $0.1 \AA$, a distinct maximum of diffuse intensities was observed between 0.6 and $0.7 \AA$ (cf. Fig. 2). A rough estimate from $I \simeq$

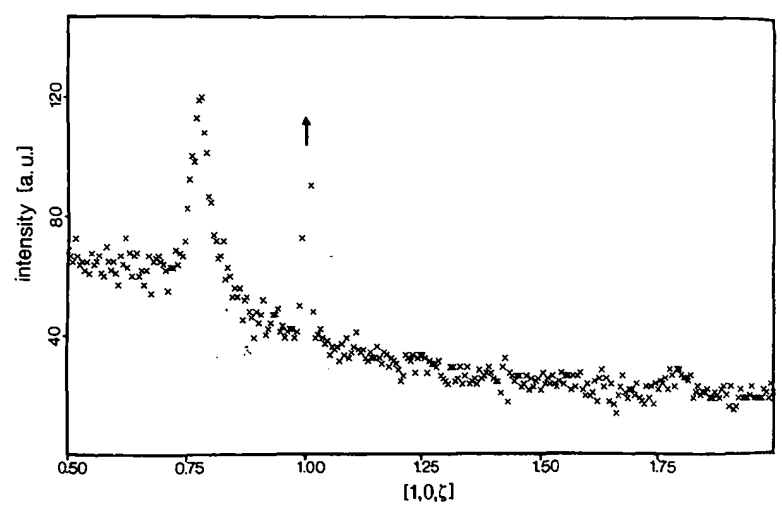

Fig. 1. Scan along [10ל] on the five-circle diffractometer (HASYLAB), $\lambda=0.7 \AA$; counting time per point: $4 \mathrm{~s}$. The peak marked by an arrow is a Bragg reflection with peak height exceeding the frame of the plot by at least a factor of 400 . $\lambda^{3} \exp \{-\mu(\lambda) d\} \quad(d=0.4 \mathrm{~mm}) \quad$ using Victoreen's formula for $\mu(\lambda)$ (International Tables for $X$-ray Crystallography, 1962) gives $\lambda_{\text {opt }}=0.55 \AA$. The diffuse layers were completely obscured by fluorescence scattering using $\lambda \geq 1.2 \AA$, e.g. the standard wavelength $\mathrm{Cu} K \alpha$.

Usually, it is assumed that relaxed resolution $\Delta Q$ is tolerable in diffuse scattering work or even highly desirable in order to integrate over a larger volume in reciprocal space. This is valid only in cases where the diffuse intensity is smoothly varying in all three dimensions and under this aspect there would be no need to perform an experiment at the synchrotron source. The argument is invalid, however, when highly anisotropic diffuse phenomena are studied. High resolution has two basic advantages in this context. (i) Different disorder models may be distinguished from one another and (ii) complicated resolution corrections may be avoided. The main consequence is to keep the wavelength dispersion $\Delta \lambda$, i.e. the radial component $\Delta Q_{\|}$of $\Delta \mathbf{Q}$, as low as possible.

The resolution of a focusing $\mathrm{Ge}(111)$ monochromator on a rotating anode (Mo) was $\Delta \lambda / \lambda=6$ $\times 10^{-3}$. Plane perfect monochromator crystals $\mathrm{Ge}(111)$ were used at the storage ring:

$$
\begin{aligned}
\Delta \lambda / \lambda & =\Delta \tau / \tau+\tan \theta_{M}\left(\alpha^{2}+\eta_{M}^{2}\right)^{1 / 2} \\
& \simeq \cot \theta_{M}\left(\Delta \theta_{\mathrm{dyn}}+\alpha\right) \\
& \simeq 3.8 \times 10^{-4} \\
\Delta \theta_{\mathrm{dyn}} & =\tan \theta_{M}(4 / \pi) r_{e} d^{2}|F|^{2} / a^{3} .
\end{aligned}
$$

$\alpha$ denotes the primary divergence of the beam, $a$ the lattice constant, $r_{e}$ the electron radius, the monochromator mosaic $\eta_{M}$ is known to be negligibly small, $d$ and $F$ have their usual meanings.

The components $\Delta Q_{\perp}$ are mainly affected by the size and mosaicity of the sample and by the collimation angles. We consider, as an example, the diffuse layer at $l=0.78$ where the effect of wavelength dispersion is negligible - at least for small $h, k$ values. The experiment on the rotating anode was performed with focusing on the zeroth layer, where the influence of the crystal size is negligible (Jagodzinski, 1968). There is some defocusing at $l=$ 0.78 due to the sample mosaicity which is negligible in our case $\left(\eta_{M} \approx 1^{\prime}\right)$. A further contribution comes from the 'acceptance' angle of the monochromator which was $15 \mathrm{mrad}$. On the other hand, for the synchrotron, $\Delta Q_{\perp}$ is determined by the very low beam divergence as long as no focusing elements are used; the high resolution is not restricted to one specific section through reciprocal space.

In K-hollandite, it is a crucial point to decide between fluid-like behaviour of the channel ions or a disordered structure with more or less pronounced 
long-range order. The FWHM of the 101 Bragg reflection measured along $c^{*}$ was $0.005 c^{*}$ (synchrotron) compared with $0.022 c^{*}$ (laboratory). The FWHM of the diffuse layer at $l=0.78$ was 0.042 $\pm 0.003 c^{*}$ (synchrotron) and 0.052 $\pm 0.011 c^{*}$ (laboratory). This is a clear indication of fluid-like behaviour of the channel ions. The correlation length

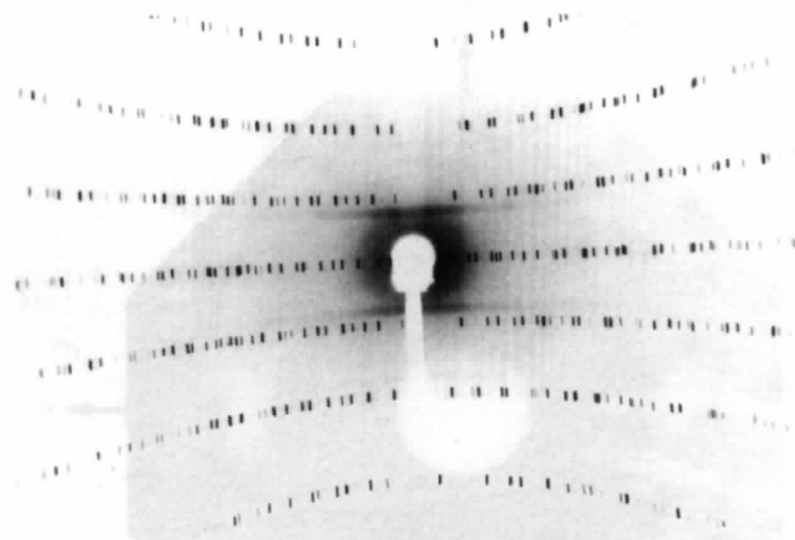

(a)

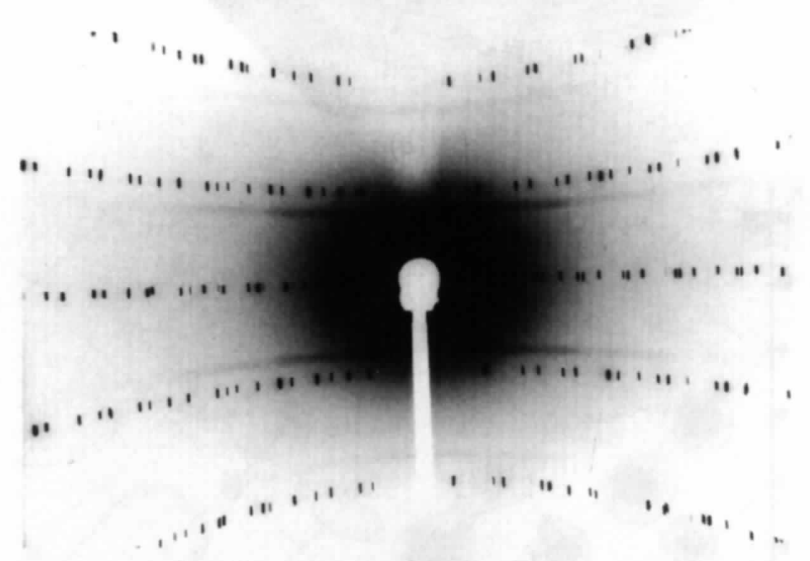

(c)

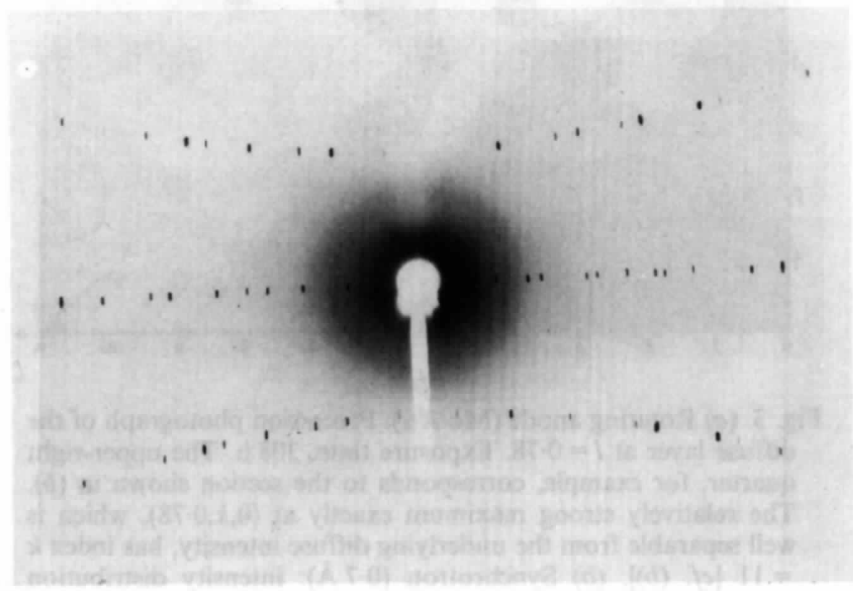

(e) within a chain is easily determined to $23 \pm 1 c$. The profile across the first layer may be interpreted directly as the Fourier transform of a correlation function between next-nearest neighbours within short-range-order models. The absolute position of the diffuse layer could be determined to 0.782 with an accuracy of $\pm 0.0002 c^{*}$ (Fig. 1). This high preci-

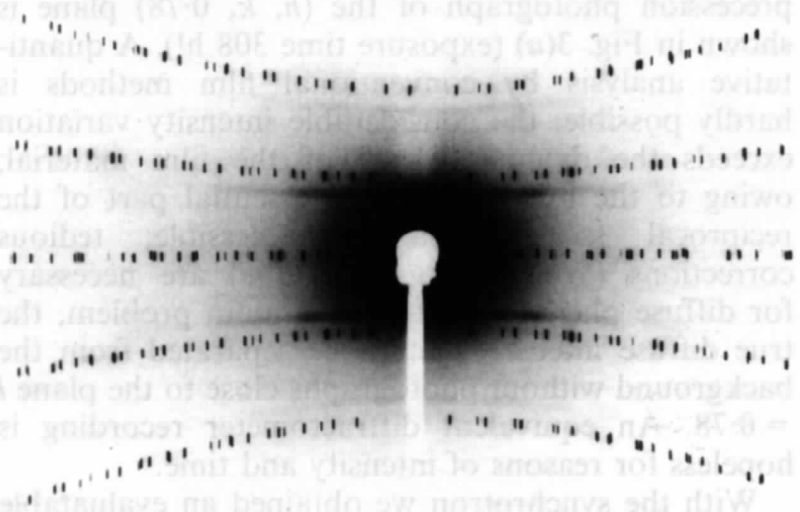

(b)

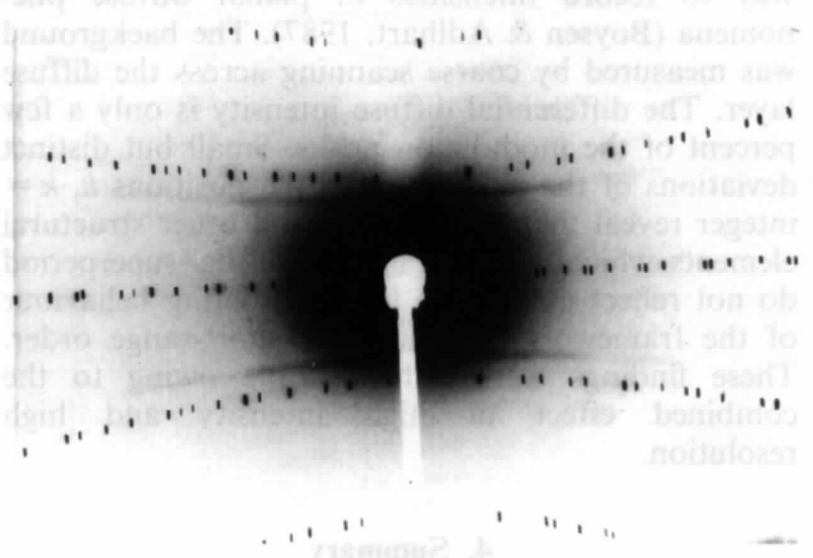

(d)

Fig. 2. Oscillation photographs $\left( \pm 55^{\circ}\right)$ taken with synchrotron radiation with different wavelengths. Exposure times between 1 and $2 \mathrm{~h}$, but scaled in the figure allowing for a rough visual comparison. $(a) \lambda=0.5,(b) 0.6,(c) 0.7,(d) 0.8$ and $(e) 1.0 \AA$. 
sion facilitates the discussion of commensurability effects. In K-hollandite it is unknown at the present stage whether or not an irreversible change to a fivefold superstructure along the chain direction occurs during cooling (Rosshirt, 1988).

To compare the intensity of the different diffuse layers along $\mathbf{c}^{*}$, one has to consider the modulations, otherwise 'wrong' intensities are interpreted. A precession photograph of the $(h, k, 0.78)$ plane is shown in Fig. 3(a) (exposure time $308 \mathrm{~h}$ !). A quantitative analysis by conventional film methods is hardly possible: the considerable intensity variation exceeds the dynamic range of the film material; owing to the blind region, an essential part of the reciprocal space remains inaccessible; tedious corrections (Weissenberg technique) are necessary for diffuse phenomena; and, the main problem, the true diffuse intensity cannot be separated from the background without photographs close to the plane $l$ $=0 \cdot 78$. An equivalent diffractometer recording is hopeless for reasons of intensity and time.

With the synchrotron we obtained an evaluatable diagram in $12 \mathrm{~h}$ by pointwise scanning within the diffuse plane (Fig. $3 b$ ). This is by no means the best way to record intensities of planar diffuse phenomena (Boysen \& Adlhart, 1987). The background was measured by coarse scanning across the diffuse layer. The differential diffuse intensity is only a few percent of the modulation peaks. Small but distinct deviations of the maxima from the positions $h, k=$ integer reveal that the $\mathrm{K}^{+}$ions and other structural elements which are responsible for this superperiod do not reflect merely the lateral ordering behaviour of the framework in a kind of short-range order. These findings were only possible owing to the combined effect of high intensity and high resolution.

\section{Summary}

Previous studies of diffuse scattering with synchrotron radiation have been extended with respect to highly anisotropic and - partly - very weak diffuse phenomena. The main advantage of a synchrotron facility is the high resolution over a wide $Q$ range, which is necessary to decide between different disorder models, in contrast to studies of local atomic arrangements, where usually only small parts of the reciprocal space have to be measured. Of great importance is the possibility of selecting an optimum wavelength to adjust the best contrast. A real shortcoming for studying diffuse scattering is the usual instrumental background. The intensity gain at the synchrotron is necessary for a quantitative study of diffuse intensities which are too weak to be recorded by conventional methods in a laboratory in a reasonable time.
In a dedicated instrument, the intensity may even be increased by a large focusing angle parallel to the orbit plane. A tunable monochromator unit should be used in order to adjust the wavelength to the specific problem and to adapt the resolution volume to position and extension of the diffuse scattering under investigation. For a small Bragg angle, the

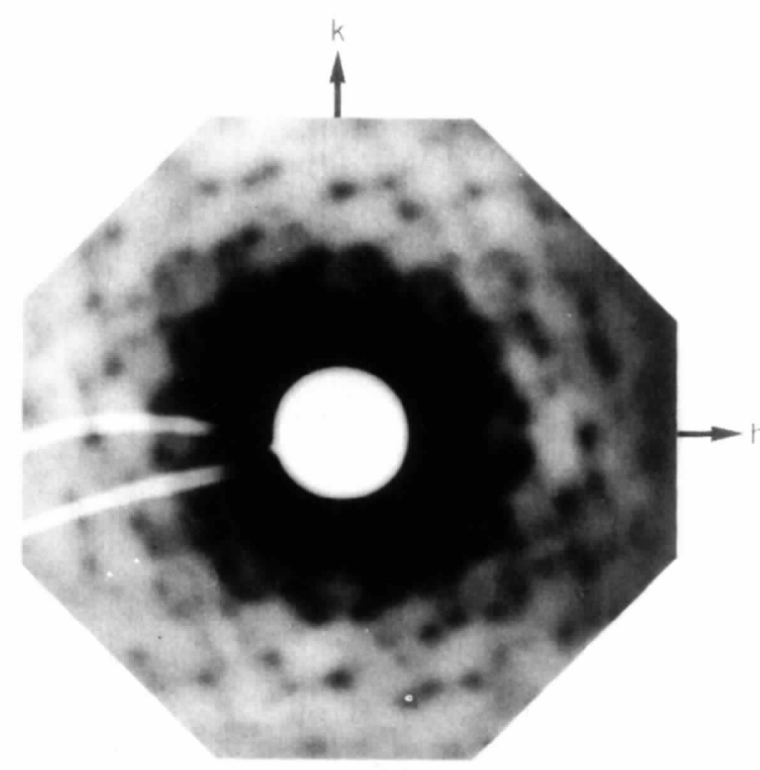

(a)

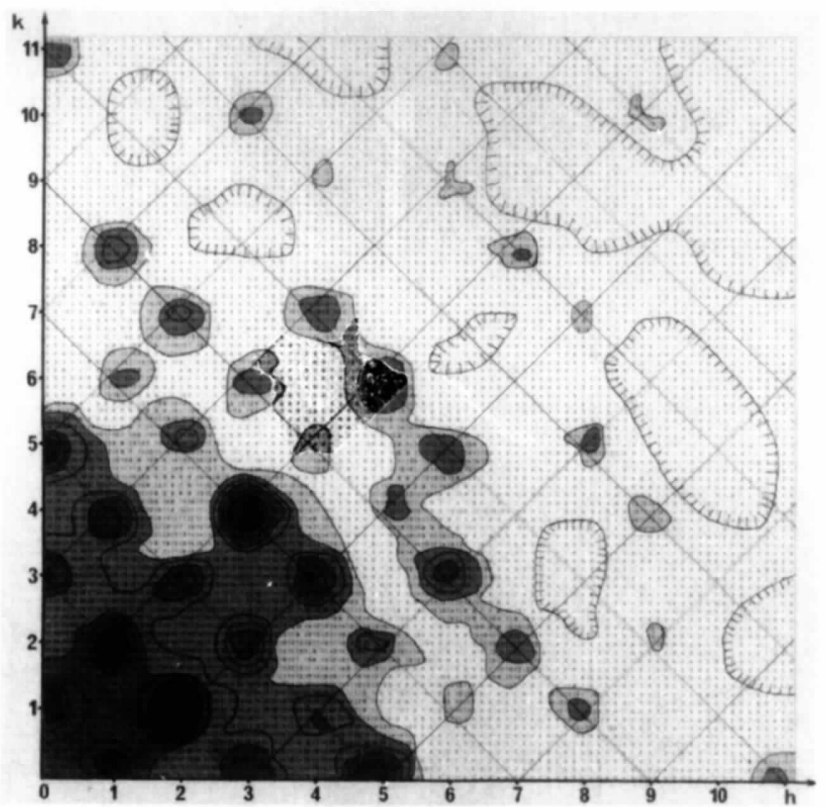

(b)

Fig. 3. (a) Rotating anode (Mo $K \alpha$ ): Precession photograph of the diffuse layer at $l=0.78$. Exposure time: $308 \mathrm{~h}$. The upper-right quarter, for example, corresponds to the section shown in (b). The relatively strong maximum exactly at $(0, k, 0 \cdot 78)$, which is well separable from the underlying diffuse intensity, has index $k$ $=11[c f .(b)]$. (b) Synchrotron $(0 \cdot 7 \AA)$ : intensity distribution within the same layer. Counting time per point: $5 \mathrm{~s}$ (total: $12 \mathrm{~h}$ ). 
intensity loss due to the polarization effect would be tolerable. With respect to the background problem, it must be emphasized that, in the presence of slowly varying diffuse phenomena [cf. Fig. $3(b)$, homogeneous part], the usual method of performing an empty scan (without sample) and subtracting the parasitic intensity afterwards is - in many cases neither practicable (low intensities) nor reliable (systematic errors). For problems of this kind which are discussed here, an evacuated sample chamber is highly recommended. The formidable resolution of the dedicated instrument can be used for the study of inhomogeneous sample materials on a fine scale. Scanning over the sample with a very narrow focused beam gives information about different stages of order. Even the time dependence of diffuse phenomena might be studied in real-time experiments: change of anisotropy, coarsening, migration of interfaces $e t c$.

This work has been funded by the German Federal Minister for Research and Technology (BMFT) under contract no. O3-SC1-LMU.

\section{References}

Boysen, H. \& Adlhart, W. (1987). J. Appl. Cryst. 20, 200-209.

International Tables for X-ray Crystallography (1962). Vol. III. Birmingham: Kynoch Press. (Present distributor Kluwer Academic Publishers, Dordrecht.)

JAGODZINSKi, H. (1968). Acta Cryst. B24, 19-23.

KupciK, V., Wendschuh-Josties, M., Wolf, A. \& Wulf, R. (1986). Nucl. Instrum. Methods Phys. Res. A246, 624-626.

KuPCIK, V., Wulf, R., Wendschuh, M., Wolf, A. PäHLER, A. (1983). Nucl. Instrum. Methods Phys. Res. 208, 519-522.

Matsubara, E. \& Georgopoulos, P. (1985). J. Appl. Cryst. 18, 377-383.

Ohshima, K., Harada, J., Morinaga, M., Geogopoulos, P. \& CoHen, J. B. (1986). J. Appl. Cryst. 19, 188-194.

Rosshirt, E. (1988). PhD thesis, LMU München, Federal Republic of Germany.

Rosshirt, E., Frey, F. \& Boysen, H. (1988). Physica (Utrecht), B156/157, 109-111.

Rosshirt, E., Frey, F., Boysen, H., Eckhold, G. \& Steigenberger, U. (1988). Mater. Sci. Forum, 27/28, 129-134. 